\title{
The modulation of collagen fibril assembly and its structure by decorin: An electron microscopic study*
}

\author{
Shunsuke Iwasaki ${ }^{1}$, Yoshinao Hosaka ${ }^{1}$, Tomohito Iwasaki ${ }^{2}$, Katsuhiro Yamamoto ${ }^{2}$, \\ Aya Nagayasu' ${ }^{1}$, Hiromi Ueda ${ }^{1}$, Yasuo Kokai $^{3}$, and Kazushige Takehana ${ }^{1}$ \\ ${ }^{1}$ Department of Veterinary Anatomy, School of Veterinary Medicine, and ${ }^{2}$ Department of Applied Biochemistry, \\ School of Dairy Science, Rakuno Gakuen University, Ebetsu; and ${ }^{3}$ Department of Biomedical Engineering, \\ School of Medicine, Sapporo Medical University, Sapporo, Japan
}

Summary. The present study was carried out to determine the effect of decorin in the process of collagen assembly. Collagen fibrils were obtained in vitro by aggregation from commercialized acid-soluble type I collagen with the addition of different concentrations of decorin (0-25 $\mu \mathrm{g} /$ $\mathrm{m} l$ ). All specimens were observed by scanning electron microscopy (SEM) and transmission electron microscopy (TEM). The distribution of collagen fibril diameters was also analyzed by TEM. In samples without or with low concentrations of decorin, highly porous collagen fiber networks were formed. On the other hand, dense networks were observed in samples treated with high concentrations of decorin. The influence of decorin secreted by cells on collagen fibrils was observed by SEM, and the fiber network elasticity was measured using a rheometer. SEM images showed that collagen fiber networks without fibroblasts were much looser than those cultured with normal fibroblasts. The networks cultured with the

Received November 20, 2007; revised March 7, 2008

* This study was supported in part by a Grant-in Aid for Scientific Research from Ministry of Education, Sports and Culture, Japan to Y.H. (No. 19780220) and by a grant from the Akiyama Foundation, Japan to Y.H. (No. 200518).

Address for correspondence: Dr. Yoshinao Hosaka, Department of Veterinary Anatomy, Faculty of Agriculture, Tottori University, 4-101 Koyama-Minami, Tottori 680-8553, Japan

Tel and Fax: +81-857-31-5572

E-mail:y-hosa@muses.tottori-u.ac.jp fibroblasts were composed of straight fibers with large diameters. On the other hand, collagen fiber networks cultured with siRNA-decorin-transfected (siDT) fibroblasts had loose, meandering fibers with small diameters. The elasticity of collagen fiber networks cultured with untransfected fibroblasts showed no significant difference over the 7-day incubation period. However, significantly lower elastic values were obtained for collagen fiber networks treated with siDT cells on days 3 and 7 . In addition, after treatment with 5.0 or $25 \mu \mathrm{g} / \mathrm{m} l$ decorin, the collagen fiber networks cultured with siDT cells exhibited an altered structure that showed a dense structure similar to that of the fiber networks cultured with untransfected fibroblasts. In conclusion, this in vitro study showed that decorin is a regulatory and architecturally small leucinerich repeat proteoglycan in the process of collagen fibril assembly.

\section{Introduction}

Connective tissues, such as the dermis, bone, tendons and ligaments, are primarily composed of collagen fibers, which are formed by type I collagen. There are also various non-collagenous proteins - proteoglycans (PGs), and glycosaminoglycans (GAGs) - which interact with the fibrillar collagen network. Many studies have suggested that interactions between collagens, PGs, and/ or GAGs play an important role in the process of collagen fibrogenesis and assembly.

Decorin, a small leucine-rich repeat PG (SLRP), is expressed by a variety of cells, including fibroblasts, myocytes, and smooth muscle cells (Thieszen and 
Roesenquist, 1995; Hocking et al., 1998, Nishimura et al., 2003). In connective tissue, it is thought that decorin participates in the regulation of collagen fibrogenesis by binding collagen fibrils (Bidanset et al., 1992; Kresse et al., 1997; Iozzo et al., 1999) in cooperation with other SLRPs and a variety of growth factors (Yamaguchi et al., 1990; Hildebrand et al., 1994; Grinnell et al., 2000; Hosaka et al., 2005). Sini et al. (1997) performed a morphological study that showed the role of decorin in the in vitro fibrogenesis of type I collagen and concluded that decorin exerted different effects on phases of fibril formation in correlation with the degree of glycosylation of the collagen. Therefore, decorin might influence the assembly and diameter of collagen fibrils by binding to their surfaces.

Several independent studies using materials such as chicken embryos and mouse tendons have suggested that decorin delays individual collagen molecule fusion (Vogel et al., 1984; Vogel and Trotter, 1987; Birk et al., 1995, 1996). It has also been shown that decorin determines the interfibrillar space of collagen fibrils by regulating the length of side chains (Kuwaba et al., 2001, 2002; Watanabe et al., 2005, 2007). Decorin also acts as an intermediary between type I collagen and some types of collagen (Nareyeck et al., 2004).

Gene-targeting studies using decorin-deficient mice have shown that SLRPs are involved in the determination of the structural phenotype of collagen fibrils and connective tissue function (Danielson et al., 1997). Decorin-deficient mice have fragile skin characterized by markedly reduced tensile strength and a thinner dermis than normal (Danielson et al., 1997; Zhang et al., 2006). Decorin deficiency also causes variation in collagen diameters in vivo, changing the mean and distribution profile of fiber diameters. Collagen alterations are observed throughout the connective tissue in decorin knockouts. Thus, decorin-knockout animals are useful for investigating decorin function in the whole body, though there are technical limitations in revealing the molecular event and mechanism of decorin in collagen fibrogenesis and its detailed assembly process in vivo. Despite these reports, much of the relationship between decorin and collagen structure has remained unknown. Especially, the relationship between decorin concentration and collagen fibrogenesis has not been fully determined.

In this study, we investigated the effect of decorincollagen fibril interaction on collagen fibril assembly and its structure using type I collagen gel, decorin, and siRNA-decorin-transfected (siDT) cells. Collagen fibrils with or without decorin were examined by electron microscopy to determine how they were affected by treatment with decorin. The level of elasticity of collagen gels was measured to evaluate their mechanical strength, and the relationship between their structure and this property was discussed.

\section{Materials and Methods}

The procedures used in the experiments are summarized in Figure 1.

\section{Preparation of type / collagen gels}

A commercially available kit (Cellmatrix type I-A, Nitta Gelatin, Osaka) of acid-soluble type I collagen gels was used for the reconstruction of collagen fibrils. This kit includes acid-soluble type I collagen, a $10 \times$ minimum essential medium (MEM) and a reconstitution buffer (50 $\mathrm{mM} \mathrm{NaOH}, 250 \mathrm{mM} \mathrm{NaHCO} 3$ and $200 \mathrm{mM}$ HEPES). Type I collagen gels were prepared according to the manufacturer's protocols. Briefly, Cellmatrix Type I-A ( 8 volumes) and $10 \times$ MEM ( 1 volume) were mixed at $4{ }^{\circ} \mathrm{C}$ and then the reconstitution buffer ( 1 volume) was added. Various concentrations of decorin $(0.1,1.0,5.0$, 10 and $25 \mu \mathrm{g} / \mathrm{m} l$; Sigma-Aldrich, St. Louis, MO, USA) were immediately added to the collagen gel mixture, and $1 \mathrm{~m} l$ of the solution was poured into each well of a 12 -well plate. The reconstructed collagen gel mixture was allowed to set over a period of $30 \mathrm{~min}$ at $37^{\circ} \mathrm{C}$. For the examination of gel contraction, some gels were released from the well wall and left to float in Dulbecco's modified Eagle's medium (DMEM) supplemented with $100 \mathrm{units} / \mathrm{m} l$ of penicillin and $100 \mu \mathrm{g} / \mathrm{m} l$ of streptomycin (Sigma-Aldrich) over a period of 7 days.

\section{siRNA transfection}

siRNA-decorin-transfected (siDT) cells were produced by suppressing the gene for decorin in mouse NIH3T3 fibroblasts using RNAi. siRNA was derived from the coding sequence of the mouse decorin gene (Genbank accession number: NM7833). siRNA selection was based on the program B-Bridge Japan (Tokyo). The target sequences and siRNA duplexes for these siRNAs were designated as siRNA (targeted sequence: 5'-AAG AGA GGC UUA UUU GAC UUC-3', sense: 5'-GAG AGG CUU AUU UGA CUU CdTdT-3', antisense: 5'-GAA GUC AAA UAA GCC UCU CdTdT-3'). 
Experiment design for decorin knockdown in $\mathrm{NIH} 3 \mathrm{T3}$ cells using siRNA

Two days before making the gel and cell mixture, $5 \times 10^{4}$ cells/well were plated in a six-well plate. The following day, one batch of the cells was transfected with GeneEraser (Stratagene, La Jolla, CA, USA) containing $10 \mathrm{nM}$ siRNA in fetal bovine serum-free DMEM. The following day, siRNA-treated or untreated NIH3T3 cells were trypsinized and mixed with the gel solution (cellgel mixture), and $1 \mathrm{~m} l$ of the mixture was poured into each well of a 12-well plate (final concentration: $5 \times 10^{4}$ cells $/ \mathrm{m} l$ ). Each well wall was coated with $3 \%$ poly-2hydroxyethl methacryate (Sigma-Aldrich) in $95 \%$ ethanol to eliminate any adhesion of cells to the growth well surfaces. Some of the cell-gel mixture was poured into glass dishes ( $1 \mathrm{~m} l$ of mixture per $17 \mathrm{~mm}$ diameter dish) for rheological analyses. After the setting of the cell-gel mixture, the culture medium was added to the gels and changed every 3 days. The cell-gel mixture was sampled on days 15 and 27 after commencing culture in the threedimensional environment. The cell-gel mixtures were also examined on days 1,3 , and 7 for gel elasticity. After the setting of the cell-gel mixture, treatment with siRNA was performed every 6 days, and some groups were treated with 5.0 or $25 \mu \mathrm{g} / \mathrm{m} l$ decorin instead of siRNA on day 18 and 24. Specimens were collected on day 27.

\section{Sample preparation for ultrastructural analysis by scanning electron microscopy and transmission electron microscopy}

Collected specimens were prepared for ultrastructural analyses by scanning electron microscopy (SEM) or transmission electron microscopy (TEM). After rinsing with a phosphate buffer, collagen gels were fixed in $3 \%$ glutaraldehyde in a $0.1 \mathrm{M}$ phosphate buffer $(\mathrm{pH}$ 7.4) for $1 \mathrm{~h}$ at $4^{\circ} \mathrm{C}$, and then samples were rinsed with distilled water, post-fixed in $1 \%$ osmium tetraoxide for $1 \mathrm{~h}$ at room temperature, rinsed again with distilled water, and dehydrated in a graded concentration of ethanol. For SEM observation, the specimens were dried by the $\mathrm{t}$ butyl alcohol freeze-drying method (Inoue and Osatake, 1988), mounted on metal stubs, coated with osmium using an osmium coater (Eiko IB3; Ibaraki), and observed

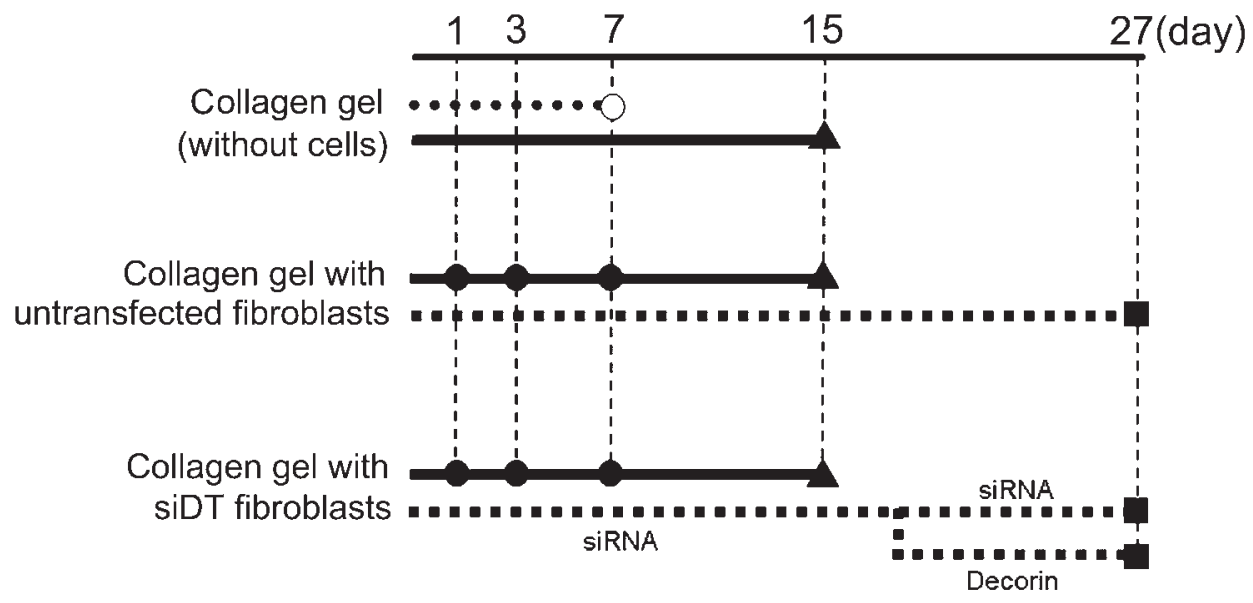

Fig. 1. Summary procedures used in the experimental procedures. Reconstructed collagen gel mixtures with various concentrations of decorin were incubated for 7 days and observed under an electron microscope (O). Specimens of cell-gel mixtures were sampled on days 1, 3, and 7 for measurement of the gel elasticity (1) and for SEM observation of the morphology of collagen fibrils (incubated for 15 days) (A). SiRNA treatment demonstrated every 6 days and incubated for 27 days. In some specimens, 5.0 or $25 \mu \mathrm{g} / \mathrm{m} l$ of decorin was added instead of siRNA from day 18 onwards. These specimens were collected for SEM observation (ם). 

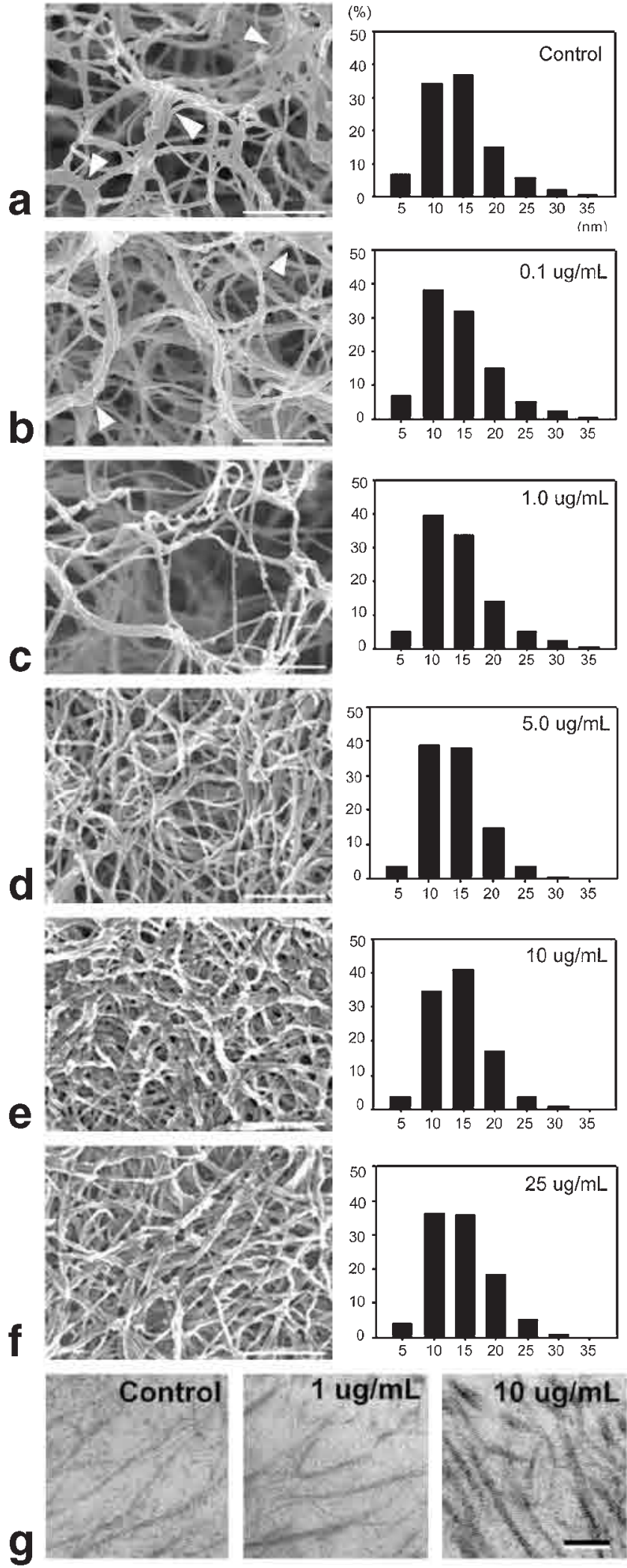

under a scanning electron microscope (JSM-6000F, JEOL, Tokyo) with an accelerating voltage of $3 \mathrm{kV}$. For TEM observation, the samples were embedded in resin (Quetol 812). Ultra-thin (110 nm in thickness) sections were mounted on a copper grid, stained with $1 \%$ uranyl acetate for $8 \mathrm{~min}$ and $1 \%$ lead citrate for an additional $10 \mathrm{~min}$, and examined by transmission electron microscope (JEM-1200, JEOL) with an accelerating voltage of $80 \mathrm{kV}$. For measurements of collagen fibril diameter and area of collagen fibrils per unit, 500 fibrils were randomly selected in photographs of each collagen gel. Independent experiments were carried out three times.

\section{Rheological analysis and measurement of gel elasticity}

A spherical plunger of $7 \mathrm{~mm}$ in diameter was stabbed into the gels at a rate of $0.5 \mathrm{~mm} / \mathrm{sec}$ using a creep meter (RE-33005, Yamaden, Tokyo). The apparent elasticity of the collagen gels was calculated from the load versus indentation curve obtained using an equation based on Hertz's theory (Lee and Radok, 1960):

$$
F=(16 \sqrt{R} / 3) \times G \times 1.5 h,
$$

where $F$ is the load $(N), R$ is the radius of the plunger ( 3.5 $\mathrm{mm}), \mathrm{G}$ is apparent elasticity $(\mathrm{Pa})$, and $\mathrm{h}$ is the indentation $(0.7 \mathrm{~mm})$.

\section{Statistical analysis}

One-way analysis of variance (ANOVA) followed by Fisher's test using Statview $($ for Windows, version 5.0, was applied for analyses of the area of collagen fibril per unit, and Student's $t$-test, Microsoft Office Excel 2003 applied rheological analysis. In both results, significant difference was set at $P<0.05$.
Fig. 2. SEM images and histograms of the diameter of collagen fibrils in a collagen fiber network. Collagen fiber network treated with 0 (control, a), $0.1 \mu \mathrm{g} / \mathrm{m} l$ (b), $1.0 \mu \mathrm{g} / \mathrm{m} l$ (c), $5.0 \mu \mathrm{g} /$ $\mathrm{m} l(\mathbf{d}), 10 \mu \mathrm{g} / \mathrm{m} l(\mathbf{e})$, and $25 \mu \mathrm{g} / \mathrm{m} l$ (f) of decorin. Membranous structures (arrowheads) appear in the fiber network treated with low concentrations $(0$ and $0.1 \mu \mathrm{g} / \mathrm{m} l)$. Bar $=1 \mu \mathrm{m}$. g: TEM images of collagen fibrils in a collagen fiber network treated with decorin. Bar $=100 \mathrm{~nm}$. 


\section{Results}

\section{Effects of decorin on collagen assembly and fibril diameter}

Highly porous collagen fiber networks were formed without decorin (control) or with low concentrations of decorin $(0.1$ or $1.0 \mu \mathrm{g} / \mathrm{m} l$ ) (Fig. $2 \mathrm{a}-\mathrm{c}$ ). Membranous structures were often observed in these fiber networks. In contrast, the addition of $5.0 \mu \mathrm{g} / \mathrm{m} l$ decorin tightened the mesh network, and similar dense fiber networks were also produced in the samples treated with 10 or $25 \mu \mathrm{g} /$ $\mathrm{m} l$ decorin (Fig. 2d-f). Membranous structures were not found in these fiber networks. Furthermore, the gels treated with high concentrations of decorin were not released from the well walls, indicating that the gel did not contract in these samples. The unreleased the gel was still adhered to the well walls at the end of the incubation period (day 7). Between the released gels and unreleased gels, collagen fibril assembly did not appear to differ at any concentration (data not shown).

The average collagen fibril diameter was unchanged regardless of the concentration of decorin, and the median collagen fibril diameter was similar in all specimens. However, the range of collagen fibril diameters in the fiber networks was smaller in the samples treated with a high concentration of decorin than in the samples treated with decorin at a concentration less than $1.0 \mu \mathrm{g} / \mathrm{m} l$ (Fig. 2, Table 1). The densities of collagen fibrils (percentage to occupation rate of collagen fibrils in cross section fields) were obtained from TEM micrographs. These values in the fiber networks without decorin (control) and with decorin $(0.1,1.0,5.0,10,25 \mu \mathrm{g} / \mathrm{ml})$ were $9.0 \pm 0.3$, $10.3 \pm 1.3,9.6 \pm 1.0,16.7 \pm 1.0,16.3 \pm 1.5$, and $16.5 \pm 1.4$, respectively. Significantly high densities of collagen fibrils $(\%)$ were observed in the samples treated with $5.0 \mu \mathrm{g} / \mathrm{m} l$ or more of decorin as compared with the density in controls (Fig. 2, Table 1).

\section{Effect of decorin on collagen fibrils and fiber network elasticity}

SEM images showed that collagen fiber networks without fibroblasts were much looser (Fig. 3a) than those cultured with untransfected fibroblasts (Fig. 3b). The networks cultured with untransfected fibroblasts were composed of almost straight fibers with large diameters. On the other hand, collagen fiber networks cultured with siDT fibroblasts were loose and composed of collagen fibers with small diameters (Fig. 3c). The elasticity of collagen fiber networks with untransfected fibroblasts showed no significant difference over the incubation period. In contrast, on days 3 and 7 , significantly lower elastic values than that on day 1 were obtained for collagen fiber networks cultured with siDT fibroblasts (Fig. 3d).

\section{Recovery of collagen fibril assembly by addition of decorin after decorin knockdown}

Collagen fiber networks cultured with siDT fibroblasts (Fig. 4b) were much looser than networks cultured with untransfected fibroblasts (Fig. 4a). After collagen fiber networks cultured with siDT fibroblasts had been supplemented with 5.0 or $25 \mu \mathrm{g} / \mathrm{m} l$ decorin, the structure of the fiber networks changed from loose to dense and became similar to the structure of fiber networks cultured with untransfected fibroblasts (Fig. 4c, d).

\section{Discussion}

By morphological and mechanical analyses, we found that decorin significantly influences collagen fibril assembly, diameter, and mechanical strength.

It is thought that decorin binds to the surface of collagen fibrils via its core protein and that it regulates collagen fibril morphology (Vogel and Trotter, 1987;

Table 1. Parameter of collagen fibrils

\begin{tabular}{lcccccc}
\hline Decorin concentration $(\mu \mathrm{g} / \mathrm{m} l)$ & $0(\mathrm{control})$ & 0.1 & 1.0 & 5.0 & 10 & 25 \\
\hline Mean $(\mathrm{nm})$ & $11.8 \pm 5.37$ & $11.5 \pm 5.42$ & $11.5 \pm 5.14$ & $11.5 \pm 4.44$ & $11.8 \pm 4.29$ & $11.7 \pm 4.71$ \\
Median $(\mathrm{nm})$ & 10.8 & 10.8 & 10.6 & 10.8 & 11.2 & 11.1 \\
Range $(\mathrm{nm})$ & $2.62-35.7$ & $2.62-35.9$ & $3.18-31.9$ & $2.94-28.9$ & $3.17-26.3$ & $3.16-26.8$ \\
\hline $\begin{array}{l}\text { Density of collagen fibrils } \\
\text { in cross section fields }(\%)\end{array}$ & $8.95 \pm 0.37^{a}$ & $10.3 \pm 1.33^{a}$ & $9.63 \pm 0.97^{a}$ & $16.3 \pm 1.09^{b}$ & $16.3 \pm 1.51^{b}$ & $16.5 \pm 1.15^{b}$ \\
\hline
\end{tabular}

Different letters ( $a$ and $b$ ) indicate significant differences $(P<0.05)$ 

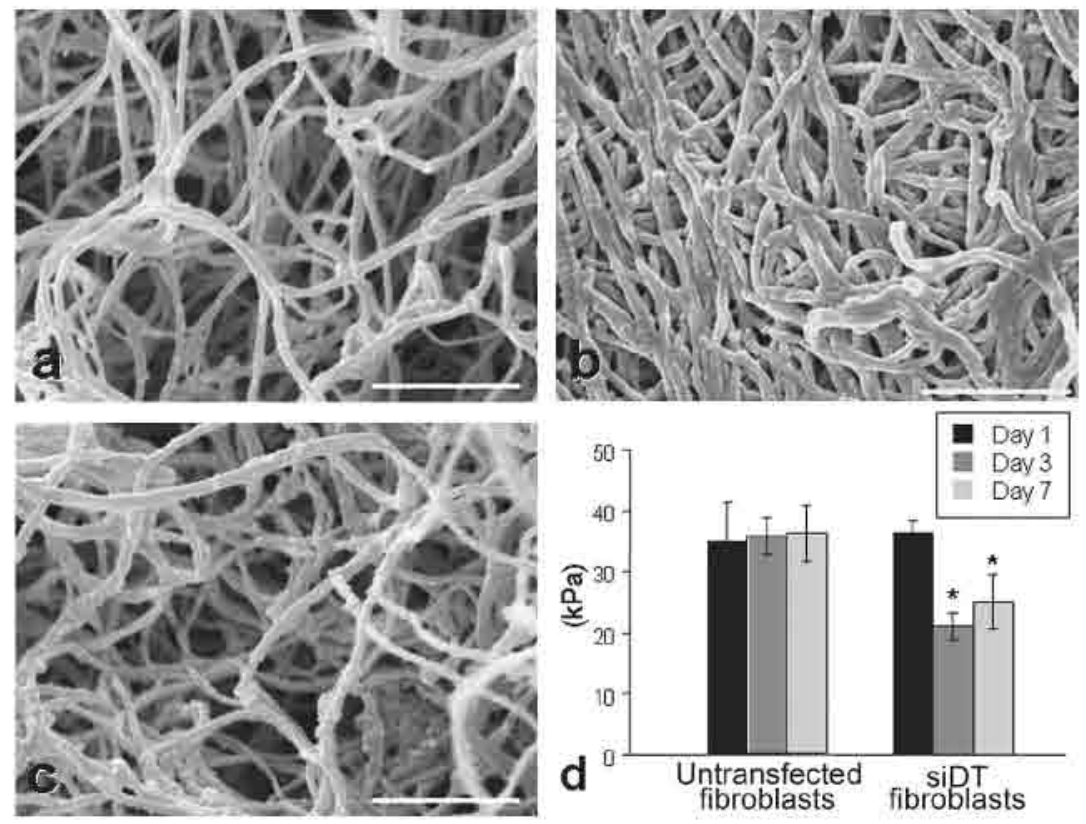

Fig. 3. SEM images of collagen fibrils on day 15 cultured without cells (gel only, a), with untransfected fibroblasts (b), and with siDT fibroblasts (c). Bar $=1 \mu \mathrm{m}$. Elasticity of the collagen fiber network cultured with untransfected fibroblasts and siDT fibroblasts (d). *: Significant difference against the value on day 1 in the same group $(P<0.05)$.
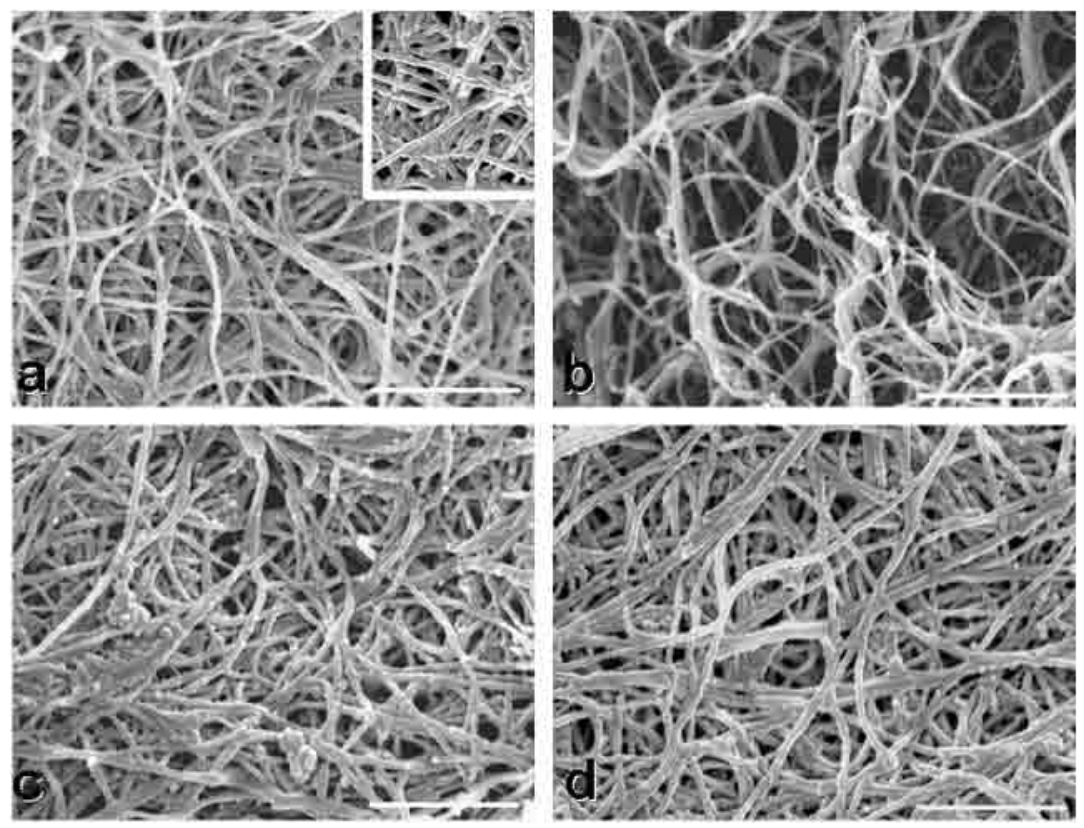

Fig. 4. Effects of decorin on recovery procedure in collagen fibril assembly. All pictures were taken on day 27 . Fiber networks cultured with untransfected fibroblasts (a) and siDT fibroblasts (b) and 5.0 (c) or $25 \mu \mathrm{g} /$ $\mathrm{m} l$ decorin (d)-treated fiber networks cultured with siDT fibroblasts. Bar $=1 \mu \mathrm{m}$. Inset in a is a micrograph of the specimen treated with only a transfection reagent without siRNA.

Bidanset et al., 1992; Scott, 1996, 2001; Raspanti et al., 2002). Decorin also plays a role in the assembly of collagen fibrils, being involved in the determination of fibril diameter (Danielson et al., 1997; Corsi et al., 2002). Our data support results of previous studies and further revealed that an increase in the amount of decorin in the process of collagen assembly results in an increase in density of the collagen fibril network. In addition, membranous structures could be seen in the fiber networks either not treated or treated with very low concentrations of decorin. These findings suggest that decorin is an essential regulatory factor in the process of collagen aggregation.

Danielson et al. (1997) demonstrated that decorindeficient animals exhibited a wider range of collagen fibril diameters than did wild-type specimens. However, our study clarified that decorin has indistinct effects in changing the average diameter of collagen fibrils. Our 
study showed that the average diameter of collagen fibrils was not significantly changed regardless of the concentration of decorin. It is generally agreed that decorin is involved in fibril formation because it inhibits an increase in collagen fibril size in vivo (Weber et al., 1996; Naeme et al., 2000; Reed and Iozzo, 2002). Our previous studies demonstrated that the density and distribution of collagen fibril diameters in a tendon, which expresses high levels of type I collagen, showed regional differences that were concomitant to the amount of decorin (Watanabe et al., 2005). However, it should be pointed out that various other factors might be involved in the regulation of collagen fibrogenesis in vivo, and further studies are needed to identify those factors.

Irregular profiles and abnormal fibril fusion patterns have been observed in vivo in decorin-deficient mice. These animals have extremely fragile skin that tears very easily (Danielson et al., 1997; Zhang et al., 2006). In our samples, the elasticity of collagen fiber networks treated with siDT fibroblasts showed significantly lower values. This might be caused by structural fragility due to a lack of decorin. Moreover, the addition of decorin (5.0 or $25 \mu \mathrm{g} / \mathrm{ml} l$ ) to the samples with siDT fibroblasts allowed collagen to adopt a normal fibril morphology. These results again indicate that decorin is the "architectural" SLRP that maintains collagen fibril configuration and provides strength of structure rheologically.

In the present study, we applied the RNAi method to control the expression of decorin in collagen fiber networks and observed drastic structural changes. Application of RNAi methods to other SLRPs may be useful for understanding the collagen fibrogenesis procedure more precisely. Moreover, suppression of decorin expression using RNAi may possibly serve toward the therapeutic treatment of some connective tissue disorders - such as scar formation - since decorin mRNA and protein are abundantly expressed and localized in scar connective tissue (Napoli et al., 1990; Lee et al., 2004). Thus, injection of decorin mRNA-suppressed cells (via decorin gene knockdown) into scar connective tissue may be useful to induce a softening of collagen tissues.

Our study was carried out three-dimensionally by SEM, whereas most previous studies were conducted twodimensionally by TEM. As Raspanti et al. (2007) recently pointed out, TEM has a higher resolution and offers an excellent depiction of detailed collagen structures. We applied TEM to measure the diameter and density of collagen fibers; however, there are limitations in the thickness of the sections (around $100 \mathrm{~nm}$ ). Under these conditions, the whole structure of the specimens above and below individual sections is difficult to determine unless serial sections are made. By using SEM, we can observe in detail the whole structure of collagen fiber networks, thickness (diameter), and degree of aggregation, fusion, and orientation of collagen fibers.

In conclusion, this in vitro study showed that decorin is a key regulatory SLRP in the process of collagen fibril assembly and collagen morphogenesis. However, further, detailed investigation is required to determine the function of each SLRP (biglycan, lumican, and fibromodulin) in the complex process of collagen morphogenesis.

\section{Acknowledgements}

The authors are grateful to Dr. Martin Grünert, Institute of Medical Biology, Singapore, for a review of this article, and to Dr. Kunio Nakamura, Department of Rheology, Rakuno Gakuen University, Ebetsu, for advice on measurement of the elasticity of the collagen gel.

\section{References}

Bidanset DJ, Guidry C, Rosenberg LC, Choi HU, Timpl $\mathrm{R}$, Hook M: Binding of the proteoglycan decorin to collagen type I. J Biol Chem 267: 5250-5256 (1992).

Birk DE, Nurminskaya MV, Zycband EI: Collagen fibrillogenesis in situ: fibril segments undergo postdeponsitional modifications resulting in linear and lateral growth during matrix development. Dev Dyn 202: 229-243 (1995).

Birk DE, Hahn RA, Linsenmayer C, Zycband EI: Characterization of collagen fibril segments from chicken embryo cornea, dermis and tendon. Matrix Biol 15: 111-118 (1996).

Corsi A, Xu T, Chen XD, Boyde A, Liang J, Mankani M, Sommer B, Iozzo RV, Eichstetter I, Robey PG., Bianco P, Young MF: Phenotypic effects of biglycan deficiency are linked to collagen fibril abnormalities, are synergized by decorin deficiency, and mimic EhlersDanlos-like changes in bone and other connective tissues. J Bone Miner Res 17: 1180-1189 (2002).

Danielson KG, Baribault H, Holmes DF, Graham H, Kadler KE, Iozzo RV: Targeted disruption of decorin leads to abnormal collagen fibril morphology and skin fragility. J Cell Biol 136: 729-743 (1997).

Grinnell F: Fibroblast-collagen-matrix contraction: growth-factor signalling and mechanical loading. Trends Cell Biol 10: 362-365 (2000).

Hildebrand A, Romaris M, Rasmussen LM, Heinegard D, Twardzik DR, Border WA, Ruoslahti E: Interaction of the small interstitial proteoglycans biglycan, decorin and fibromodulin with transforming growth factor $\beta$. Biochem J 302: 527-534 (1994). 
Hocking AM, Shinomura T, McQuillan DJ: Leucine-rich repeat glycoproteins of the extracellular matrix. Matrix Biol 17:1-19 (1998).

Hosaka Y, Kirisawa R, Mafune N, Takehana K: Downregulation of decorin and transforming growth factor- $\beta 1$ by decorin gene suppression in tendinocytes. Connect Tissue Res 46: 18-26. (2005).

Inoue T, Osatake H: A new drying method of biological specimens for scanning electron microscopy: The tbutyl alcohol freeze-drying method. Arch Histol Cytol 51: 53-59 (1988).

Iozzo RV: The biology of the small leucine-rich proteoglycans. Functional network of interactive proteins. J Biol Chem 274: 18843-18846 (1999).

Kresse H, Liszio C, Schönherr E, Fisher LW: Critical role of glutamate in a central leucine-rich repeat of decorin for interaction with type I collagen. J Biol Chem 18: 18404-18410 (1997).

Kuwaba K, Kobayashi M, Nomura Y, Irie S, Koyama Y: Elongated dermatan sulphate in post-inflammatory healing skin distributes among collagen fibrils separated by enlarged interfibrillar gaps. Biochem $J$ 358: 157-163 (2001).

Kuwaba K, Kobayashi M, Nomura Y, Irie S, Koyama $Y$ : Size control of decorin dermatan sulfate during remodeling of collagen fibrils in healing skin. $J$ Dermatol Sci 29: 185-194 (2002).

Lee HE, Radok JRM: The contact problem for viscoelastic bodies. J Appl Mech 27: 438-44 (1960).

Lee PH, Trowbridge JM, Taylor KR, Morhenn VB, Gallo RL: Dermatan sulfate proteoglycan and glycosaminoglycan synthesis is induced in fibroblasts by transfer to a three-dimensional extracellular environment. J Biol Chem 279: 48640-48646 (2004).

Naeme PJ, Kay CJ, McQuillan DJ, Beales MP, Hassell JR: Independent modulation of collagen fibrillogenesis by decorin and lumican. Cell Mol Life Sci 57: 859-863 (2000).

Napoli C, Lemieux C, Jorgensen R: Introduction of a chimeric chalcone synthase gene into petunia results in reversible co-suppression of homologous genes in trans. Plant Cell 2: 279-289 (1990).

Nareyeck G, Seidler DG, Troyer D, Rauterberg J, Kresse H, Schönherr E: Differential interactions of decorin and decorin mutants with type I and type VI collagens. Eur J Biochem 271: 3389-3398 (2004).

Nishimura T, Taneichi A, Wakamatu J, Hattori A: Effect of skeletal muscle decorin on collagen fibrillogenesis in vitro. Animal Sci J 74: 399-405 (2003).

Raspanti M, Congiu T, Guizzardi S: Structural aspects of the extracellular matrix of the tendon: an atomic force and scanning electron microscopy study. Arch Histol Cytol 65: 37-43 (2002).

Raspanti M, Viola M, Sonaggere M, Tira ME, Tenni $\mathrm{R}$ : Collagen fibril structure is affected by collagen concentration and decorin. Biomacromolecules. 8: 2087-2091(2007).

Reed CC, Iozzo RV: The role of decorin in collagen fibrillogenesis and skin homeostasis. Glycoconj $J 19$ : 249-255 (2002).

Scott JE: Proteodermatan and proteokeratan sulfate (decorin, lumican/fibromodulin) proteins are horseshoe shaped. Implications for their interactions with collagen. Biochemistry 35: 8795-8799 (1996).

Scott JE: Structure and function in extracellular matrices depend on interactions between anionic glycosaminoglycans. Pathol Biol 49: 284-289 (2001).

Sini P, Denti A, Tira ME, Balduini C: Role of decorin on in vitro fibrillogenesis of type I collagen. Glycoconj $J$ 14: 871-874 (1997).

Thieszen SL, Rosenquist TH: Expression of collagens and decorin during aortic arch artery development: implications for matrix pattern formation. Matrix Biol 14: 573-582 (1995).

Vogel KG, Trotter JA: The effect of proteoglycans on the morphology of collagen fibrils formed in vitro. Collagen Relat Res 7: 105-114 (1987).

Vogel KG, Paulsson M, Heinegard D: Specific inhibition of type I and type II collagen fibrillogenesis by the small proteoglycan of tendon. Biochem J 223: 587-597 (1984).

Watanabe T, Hosaka Y, Yamamoto E, Ueda H, Sugawara $\mathrm{K}$, Takahashi $\mathrm{H}$, Takehana $\mathrm{K}$ : Control of the collagen fibril diameter in the equine superficial digital flexor tendon in horses by decorin. J Vet Med Sci 67: 855-860 (2005).

Watanabe T, Imamura Y, Hosaka Y, Ueda H, Takehana $\mathrm{K}$ : Graded arrangement of collagen fibrils in the equine superficial digital flexor tendon. Connect Tissue Res 48: 332-337 (2007).

Weber IT, Harrison RW, Iozzo RV: Model structure of decorin and implications for collagen fibrillogenesis. $J$ Biol Chem 271: 31767-31770 (1996).

Yamaguchi Y, Mann DM, Ruoslahti E: Negative regulation of transforming growth factor-beta by the proteoglycan decorin. Nature 346: 281-284 (1990).

Zhang G, Ezura Y, Chervoneva I, Robinson PS, Beason DP, Carine ET, Soslowsky LJ, Iozzo RV, Birk DE: Decorin regulates assembly of collagen fibrils and acquisition of biomechanical properties during tendon development. J Cell Biochem 98: 1436-1449 (2006). 